Erschienen in: Stickel, Gerhard (Hrsg.): Stilfragen. - Berlin, New York: de Gruyter, 1995. S. 378. (Institut für deutsche Sprache. Jahrbuch 1994)

\title{
Aufgaben einer modernen Stilistik
}

Vier Statements von

\author{
Birgit Stolt (Stockholm) \\ Willy Sanders (Bern) \\ Ulla Fix (Leipzig) \\ Hans Bickes (Darmstadt)
}

Siegfried Grosse (Bochum)

\section{Moderation und Redaktion der abschließenden Plenardiskussion}

\section{SIEGFRIED GROSSE}

Meine Damen und Herren,

Im Unterschied zu früheren Jahrestagungen schließen wir diesmal nicht mit einem resümierenden Referat, sondern mit einem Podium, dessen Teilnehmer in kurzen Statements ihre Sicht einer modernen Stilistik umreißen werden. Die Damen und Herren des Podiums haben vor der Tagung ihre Positionen gegeneinander abgesteckt und dankenswerterweise in Mannheim die Ergebnisse der Tagungsreferate berücksichtigt, also ihre Statements umgeschrieben und aktualisiert. Im Anschluß an die vier Kurzreferate bitten wir Sie um Ihre Teilnahme an der Diskussion. 